\title{
15 FACE NUMBERS OF POLYTOPES AND COMPLEXES
}

\author{
Louis J. Billera and Anders Björner
}

\section{INTRODUCTION}

Geometric objects are often put together from simple pieces according to certain combinatorial rules. As such, they can be described as complexes with their constituent cells, which are usually polytopes and often simplices. Many constraints of a combinatorial and topological nature govern the incidence structure of cell complexes and are therefore relevant in the analysis of geometric objects. Since these incidence structures are in most cases too complicated to be well understood, it is worthwhile to focus on simpler invariants that still say something nontrivial about their combinatorial structure. The invariants to be discussed in this chapter are the $f$-vectors $f=\left(f_{0}, f_{1}, \ldots\right)$, where $f_{i}$ is the number of $i$-dimensional cells in the complex.

The theory of $f$-vectors can be discussed at two levels: (1) the numerical relations satisfied by the $f_{i}$ numbers, and (2) the algebraic, combinatorial, and topological facts and constructions that give rise to and explain these relations. This chapter will summarize the main facts in the numerology of $f$-vectors (i.e., at level 1), with emphasis on cases of geometric interest.

The chapter is organized as follows. To begin with we treat simplicial complexes, first the general case (Section 15.1), then complexes with various Betti number constraints (Section 15.2), and finally triangulations of spheres, polytope boundaries, and manifolds (Section 15.3). Then we move on to non-simplicial complexes, discussing first the general case (Section 15.4) and then polytopes and spheres (Section 15.5).

\subsection{SIMPLICIAL COMPLEXES}

\section{GLOSSARY}

The convex hull of any set of $j+1$ affinely independent points in $\mathbb{R}^{n}$ is called a $\boldsymbol{j}$-simplex. See Chapter 13 for more about this definition, and for the notions of faces and vertices of a simplex.

A geometric simplicial complex $\Gamma$ is a finite nonempty family of simplices in $\mathbb{R}^{n}$ such that (i) $\sigma \in \Gamma$ implies that $\tau \in \Gamma$ for every face $\tau$ of $\sigma$, and (ii) if $\sigma, \tau \in \Gamma$ and $\sigma \cap \tau \neq \emptyset$ then $\sigma \cap \tau$ is a face of both $\sigma$ and $\tau$.

An abstract simplicial complex $\Delta$ is a finite nonempty family of subsets of some ground set $V$ (the vertex set) such that if $A \in \Delta$ and $B \subseteq A$ then $B \in \Delta$. (Note that always $\emptyset \in \Delta$.) The elements $A \in \Delta$ are called faces. Define the dimension of a face $A$ and of $\Delta$ itself by $\operatorname{dim} A=|A|-1 ; \operatorname{dim} \Delta=$ 
$\max _{A \in \Delta} \operatorname{dim} A$. By a $\boldsymbol{d}$-complex we mean a $\boldsymbol{d}$-dimensional complex.

With every geometric simplicial complex $\Gamma$ we associate an abstract simplicial complex by taking the family of vertex sets of its simplices. Conversely, every $d-$ dimensional abstract simplicial complex $\Delta$ can be realized in $\mathbb{R}^{n}$ for $n \geq 2 d+1$ (and sometimes less) by some geometric simplicial complex. The latter is unique up to homeomorphism, so it is correct to think of the realization map as a oneto-one correspondence between abstract and geometric simplicial complexes. We will therefore drop the adjectives "abstract" and "geometric" and speak only of a simplicial complex.

For a simplicial complex $\Delta$, let $\Delta^{i}=\{i$-dimensional faces $\}$ and let $f_{i}=\left|\Delta^{i}\right|$. The integer sequence $f(\Delta)=\left(f_{0}, f_{1}, \ldots\right)$ is called the $f$-vector of $\Delta$. (The entry $f_{-1}=1$ is usually suppressed.) The subcomplex $\Delta^{\leq i}=\bigcup_{j \leq i} \Delta^{j}$ is called the i-skeleton of $\Delta$.

A simplicial complex $\Delta$ is called pure if all maximal faces are of equal dimension. It is called $\boldsymbol{r}$-colorable if there exists a partition of the vertex set $V=V_{1} \cup \ldots \cup V_{r}$ such that $\left|A \cap V_{i}\right| \leq 1$ for all $A \in \Delta$ and $1 \leq i \leq r$. Equivalently, $\Delta$ is $r$-colorable if and only if its 1-skeleton $\Delta^{\leq 1}$ is $r$-colorable in the standard sense of graph theory. An $(r-1)$-complex that is both pure and $r$-colorable is sometimes called balanced.

For integers $k, n \geq 1$ there is a unique way of writing

$$
n=\left(\begin{array}{c}
a_{k} \\
k
\end{array}\right)+\left(\begin{array}{c}
a_{k-1} \\
k-1
\end{array}\right)+\ldots+\left(\begin{array}{c}
a_{i} \\
i
\end{array}\right)
$$

so that $a_{k}>a_{k-1}>\ldots>a_{i} \geq i \geq 1$. Then define

$$
\partial_{k}(n)=\left(\begin{array}{c}
a_{k} \\
k-1
\end{array}\right)+\left(\begin{array}{c}
a_{k-1} \\
k-2
\end{array}\right)+\ldots+\left(\begin{array}{c}
a_{i} \\
i-1
\end{array}\right)
$$

and

$$
\partial^{k}(n)=\left(\begin{array}{c}
a_{k}-1 \\
k-1
\end{array}\right)+\left(\begin{array}{c}
a_{k-1}-1 \\
k-2
\end{array}\right)+\ldots+\left(\begin{array}{c}
a_{i}-1 \\
i-1
\end{array}\right) .
$$

Also let $\partial_{k}(0)=\partial^{k}(0)=0$.

Let $\mathbb{N}^{\infty}$ denote the set of sequences $\left(n_{0}, n_{1}, \ldots\right)$ of nonnegative integers, and $\mathbb{N}^{(\infty)}$ the subset of sequences such that $n_{k}=0$ for sufficiently large $k$. We call $n \in \mathbb{N}^{(\infty)}$ a $K$-sequence if

$$
\partial_{k+1}\left(n_{k}\right) \leq n_{k-1} \quad \text { for all } k \geq 1
$$

We call $n \in \mathbb{N}^{\infty}$ an $M$-sequence if

$$
n_{0}=1 \text { and } \partial^{k}\left(n_{k}\right) \leq n_{k-1} \quad \text { for all } k \geq 2 \text {. }
$$

\section{THE KRUSKAL-KATONA THEOREM AND SOME RELATIVES}

The following basic result characterizes the $f$-vectors of simplicial complexes.

THEOREM 15.1.1 Kruskal-Katona Theorem

For $f=\left(f_{0}, f_{1}, \ldots\right) \in \mathbb{N}^{(\infty)}$ the following are equivalent: 
(i) $f$ is the $f$-vector of a simplicial complex;

(ii) $f$ is a $K$-sequence.

A simplicial complex is connected if its 1-skeleton is connected in the sense of graph theory.

\section{THEOREM 15.1.2}

For $f \in \mathbb{N}^{(\infty)}$ the following are equivalent:

(i) $f$ is the $f$-vector of a connected simplicial complex;

(ii) $f$ is a $K$-sequence and $\partial^{3}\left(f_{2}\right) \leq f_{1}-f_{0}+1$.

Theorem 15.1.1 has a generalization to colored complexes, whose statement will require some additional definitions. Fix an integer $r>0$. Then define $\left(\begin{array}{l}n \\ k\end{array}\right)_{r}$ as follows: partition $\{1, \ldots, n\}$ into $r$ subsets $V_{1}, \ldots, V_{r}$ as evenly as possible (so every subset $V_{i}$ will have $\left\lfloor\frac{n}{r}\right\rfloor$ or $\left\lfloor\frac{n}{r}\right\rfloor+1$ elements), and let $\left(\begin{array}{l}n \\ k\end{array}\right)_{r}$ be the number of $k$-subsets $F \subseteq\{1, \ldots, n\}$ such that $\left|F \cap V_{i}\right| \leq 1$ for $1 \leq i \leq r$. For $k \leq r$ every positive integer $n$ can be uniquely written

$$
n=\left(\begin{array}{c}
a_{k} \\
k
\end{array}\right)_{r}+\left(\begin{array}{c}
a_{k-1} \\
k-1
\end{array}\right)_{r-1}+\ldots+\left(\begin{array}{c}
a_{i} \\
i
\end{array}\right)_{r-k+i},
$$

where $a_{k}>a_{k-1}>\ldots>a_{i} \geq i \geq 1$. Then define

$$
\partial_{k}^{(r)}(n)=\left(\begin{array}{c}
a_{k} \\
k-1
\end{array}\right)_{r}+\left(\begin{array}{c}
a_{k-1} \\
k-2
\end{array}\right)_{r-1}+\ldots+\left(\begin{array}{c}
a_{i} \\
i-1
\end{array}\right)_{r-k+i},
$$

and let $\partial_{k}^{(r)}(0)=0$

\section{THEOREM 15.1.3}

For $f=\left(f_{0}, \ldots, f_{d-1}\right), d \leq r$, the following are equivalent:

(i) $f$ is the $f$-vector of an r-colorable simplicial complex;

(ii) $\partial_{k+1}^{(r)}\left(f_{k}\right) \leq f_{k-1}$, for all $1 \leq k \leq d-1$.

Note that for $r$ sufficiently large Theorem 15.1.3 specializes to Theorem 15.1.1.

\section{MULTICOMPLEXES AND MACAULAY'S THEOREM}

A multicomplex $\mathcal{M}$ is a nonempty collection of monomials in finitely many variables such that if $m$ is in $\mathcal{M}$ then so is every divisor of $m$. Let $f_{i}(\mathcal{M})$ be the number of degree $i$ monomials in $\mathcal{M} ; f(\mathcal{M})=\left(f_{0}, f_{1}, \ldots\right)$ is called the $f$-vector of $\mathcal{M}$.

\section{THEOREM 15.1.4 Macaulay's Theorem}

For $f \in \mathbb{N}^{\infty}$ the following are equivalent:

(i) $f$ is the f-vector of a multicomplex;

(ii) $f$ is an $M$-sequence;

(iii) $f_{i}=\operatorname{dim}_{k} R_{i}, i \geq 0$, for some finitely generated commutative graded $\boldsymbol{k}$-algebra $R=\oplus_{i \geq 0} R_{i}$ such that $R_{0} \cong \boldsymbol{k}$ (a field) and $R_{1}$ generates $R$. 
A simplicial complex can be identified with a multicomplex of squarefree monomials. Hence, a $K$-sequence is (except for a shift in the indexing) an $M$-sequence: If $\left(f_{0}, \ldots, f_{d-1}\right)$ is a $K$-sequence then $\left(1, f_{0}, \ldots, f_{d-1}\right)$ is an $M$-sequence. For this reason (and others, see e.g. Theorem 15.2.2), properties of $M$-sequences are relevant also even if one is interested only in the special case of simplicial complexes.

A multicomplex is pure if all its maximal (under divisibility) monomials have the same degree.

\section{THEOREM 15.1.5}

Let $\left(f_{0}, \ldots, f_{r}\right)$ be the $f$-vector of a pure multicomplex, $f_{r} \neq 0$. Then $f_{i} \leq f_{j}$ for all $i<j \leq r-i$.

\section{COMMENTS}

Simplicial complexes (abstract and geometric) are treated in most books on algebraic topology; see, e.g., [Mun84, Spa66]. The Kruskal-Katona theorem (independently discovered by M.-P. Schützenberger, J.B. Kruskal, G.O.H. Katona, L.H. Harper, and B. Lindström during the years 1959-1966) is discussed in many places and several proofs have appeared; see, e.g., [And87, Zie95]. Theorems 15.1.2 and 15.1.3 are from [Bjo96] and [FFK88] respectively. A Kruskal-Katona type theorem for simplicial complexes with vertex-transitive symmetry group appears in [FK96].

For Macaulay's theorem we refer to [And87, Sta96]. There is a common generalization of Macaulay's theorem and the Kruskal-Katona theorem due to Clements and Lindström; see [And87]. Theorem 15.1.5 is from [Hib89].

\subsection{BETTI NUMBER CONSTRAINTS}

\section{GLOSSARY}

The Euler characteristic $\chi(\Delta)$ of a simplicial complex $\Delta$ with $f$-vector $\left(f_{0}, \ldots\right.$, $\left.f_{d-1}\right)$ is $\chi(\Delta)=\sum_{i=0}^{d-1}(-1)^{i} f_{i}$.

The $\boldsymbol{h}$-vector $\left(h_{0}, \ldots, h_{\boldsymbol{d}}\right)$ of a $(d-1)$-dimensional simplicial complex is defined by

$$
\sum_{i=0}^{d} h_{i} x^{d-i}=\sum_{i=0}^{d} f_{i-1}(x-1)^{d-i}
$$

The corresponding $\boldsymbol{g}$-vector $\left(g_{0}, \ldots, g_{\lfloor d / 2\rfloor}\right)$ is defined by $g_{0}=1$ and $g_{i}=h_{i}-$ $h_{i-1}$, for $i \geq 1$.

The Betti number $\beta_{i}(\Delta)$ is the dimension (as a $\mathbb{Q}$-vector space) of the $i$-th reduced simplicial homology group $\widetilde{H}_{i}(\Delta, \mathbb{Q})$; see any textbook on algebraic topology (e.g. [Mun84]) for the definition. We call $\left(\beta_{0}, \ldots, \beta_{\operatorname{dim} \Delta}\right)$ the Betti sequence of $\Delta$.

The link $\ell k_{\Delta}(F)$ of a face $F$ is the subcomplex of $\Delta$ defined by $\ell k_{\Delta}(F)=\{A \in$ $\Delta \mid A \cap F=\emptyset, A \cup F \in \Delta\}$. Note that $\ell k_{\Delta}(\emptyset)=\Delta$.

A simplicial complex $\Delta$ is acyclic if $\beta_{i}(\Delta)=0$ for all $i$. 
A simplicial complex $\Delta$ is Cohen-Macaulay if $\beta_{i}\left(\ell k_{\Delta}(F)\right)=0$ for all $F \in \Delta$ and all $i<\operatorname{dim} \ell k_{\Delta}(F)$.

A simplicial complex $\Delta$ is $\boldsymbol{m}$-Leray if $\beta_{i}\left(\ell k_{\Delta}(F)\right)=0$ for all $F \in \Delta$ and all $i \geq m$.

\section{FIXED BETTI NUMBERS}

The most basic relationship between $f$-vectors and Betti numbers is the EulerPoincaré formula:

$$
\chi(\Delta)=f_{0}-f_{1}+f_{2}-\ldots=1+\beta_{0}-\beta_{1}+\beta_{2}-\ldots
$$

This is in fact the only linear one in the following complete set of relations.

\section{THEOREM 15.2.1}

For $f=\left(f_{0}, f_{1}, \ldots\right) \in \mathbb{N}^{(\infty)}$ and $\beta=\left(\beta_{0}, \beta_{1}, \ldots\right) \in \mathbb{N}^{(\infty)}$ the following are equivalent:

(i) $f$ is the $f$-vector of some simplicial complex with Betti sequence $\beta$;

(ii) if $\chi_{k-1}=\sum_{j \geq k}(-1)^{j-k}\left(f_{j}-\beta_{j}\right), k \geq 0$, then $\chi_{-1}=1$ and $\partial_{k+1}\left(\chi_{k}+\beta_{k}\right) \leq$ $\chi_{k-1}$ for all $k \geq 1$.

By putting $\beta_{i}=0$ for all $i$ one gets as a special case a characterization of the $f$-vectors of acyclic simplicial complexes, viz., $\sum_{i \geq 0} f_{i-1} x^{i}=(1+x) \sum_{i \geq 0} f_{i-1}^{\prime} x^{i}$, where $\left(f_{0}^{\prime}, f_{1}^{\prime}, \ldots\right)$ is a $K$-sequence.

\section{COHEN-MACAULAY COMPLEXES}

Examples of Cohen-Macaulay complexes are triangulations of manifolds whose Betti numbers vanish below the top dimension, in particular triangulations of spheres and balls. Other examples are matroid complexes (the independent sets of a matroid), Tits buildings, and the order complexes (simplicial complex of totally ordered subsets) of several classes of posets, e.g., semimodular lattices (including distributive and geometric lattices). Shellable complexes (see Chapters 14 and 18) are CohenMacaulay. Cohen-Macaulay complexes are always pure.

The definition of $h$-vector given in the glossary shows that the $h$-vector and the $f$-vector of a complex mutually determine each other via the formulas:

$$
h_{i}=\sum_{j=0}^{i}(-1)^{i-j}\left(\begin{array}{c}
d-j \\
i-j
\end{array}\right) f_{j-1}, \quad f_{i-1}=\sum_{j=0}^{i}\left(\begin{array}{c}
d-j \\
i-j
\end{array}\right) h_{j},
$$

for $0 \leq i \leq d$. Hence, we may state $f$-vector results in terms of $h$-vectors whenever convenient.

\section{THEOREM 15.2.2}

For $h=\left(h_{0}, \ldots, h_{d}\right) \in \mathbb{Z}^{d+1}$ the following are equivalent:

(i) $h$ is the h-vector of a $(d-1)$-dimensional Cohen-Macaulay complex;

(ii) $h$ is the h-vector of a $(d-1)$-dimensional shellable complex;

(iii) $h$ is an $M$-sequence. 
Since there are a total of $\left(\begin{array}{c}n+k-1 \\ k\end{array}\right)$ monomials of degree $k$ in $n$ variables, and by Theorems 15.1.4 and 15.2.2 the $h$-vector of a $(d-1)$-dimensional Cohen-Macaulay complex counts certain monomials in $h_{1}=f_{0}-d$ variables, we derive the inequalities

$$
0 \leq h_{i} \leq\left(\begin{array}{c}
f_{0}-d+i-1 \\
i
\end{array}\right)
$$

for the $h$-vectors of Cohen-Macaulay complexes. The lower bound can be improved for complexes with fixed-point-free involutive symmetry.

\section{THEOREM 15.2.3}

Let $h=\left(h_{0}, \ldots, h_{d}\right)$ be the $h$-vector of a Cohen-Macaulay complex admitting an automorphism $\alpha$ of order 2 , such that $\alpha(F) \neq F$ for all $F \in \Delta \backslash\{\emptyset\}$. Then

$$
h_{i} \geq\left(\begin{array}{l}
d \\
i
\end{array}\right) \quad \text { for } 0 \leq i \leq d .
$$

Consequently, $f_{d-1}=h_{0}+\ldots+h_{d} \geq 2^{d}$.

Another condition on a Cohen-Macaulay complex that forces stricter conditions on its $h$-vector is being $r$-colorable.

\section{THEOREM 15.2.4}

For $h=\left(h_{0}, \ldots, h_{d}\right) \in \mathbb{Z}^{d+1}$ the following are equivalent:

(i) $h$ is the $h$-vector of a $(d-1)$-dimensional and d-colorable Cohen-Macaulay complex;

(ii) $\left(h_{1}, \ldots h_{d}\right)$ is the $f$-vector of a d-colorable simplicial complex.

Hence in this case the $h$-vector is not only an $M$-sequence, but the special kind of $K$-sequence characterized in Theorem 15.1.3.

\section{LERAY COMPLEXES}

Examples of Leray complexes arise as follows. Let $\mathcal{K}=\left\{K_{1}, \ldots, K_{t}\right\}$ be a family of convex sets in $\mathbb{R}^{m}$, and let $\Delta(\mathcal{K})=\left\{A \subseteq\{1, \ldots, t\} \mid \bigcap_{i \in A} K_{i} \neq \emptyset\right\}$. Then the simplicial complex $\Delta(\mathcal{K})$ is $m$-Leray.

Fix $m \geq 0$, and let $f=\left(f_{0}, \ldots, f_{d-1}\right)$ be the $f$-vector of a simplicial complex $\Delta$. Define

$$
h_{k}^{*}= \begin{cases}f_{k} & \text { for } 0 \leq k \leq m-1 \\
\sum_{j \geq 0}(-1)^{j}\left(\begin{array}{c}
k+j-m \\
j
\end{array}\right) f_{k+j} & \text { for } k \geq m .\end{cases}
$$

The sequence $h^{*}=\left(h_{0}^{*}, \ldots, h_{d-1}^{*}\right)$ is the $\boldsymbol{h}^{*}$-vector of $\Delta$. The two vectors $f$ and $h^{*}$ mutually determine each other.

THEOREM 15.2.5

For $h^{*}=\left(h_{0}^{*}, h_{1}^{*}, \ldots\right) \in \mathbb{Z}^{(\infty)}$ the following are equivalent:

(i) $h^{*}$ is the $h^{*}$-vector of an $m$-Leray complex; 
(ii) $h^{*}$ is the $h^{*}$-vector of $\Delta(\mathcal{K})$ for some family $\mathcal{K}$ of convex sets in $\mathbb{R}^{m}$;

(iii)

$$
\begin{cases}h_{k}^{*} \geq 0 & \text { for } k \geq 0 \\ \partial_{k+1}\left(h_{k}^{*}\right) \leq h_{k-1}^{*} & \text { for } 1 \leq k \leq m-1 \\ \partial_{m}\left(h_{k}^{*}\right) \leq h_{k-1}^{*}-h_{k}^{*} & \text { for } k \geq m .\end{cases}
$$

\section{COMMENTS}

The Euler-Poincaré formula (due to Poincaré 1899) is proved in most books on algebraic topology. Theorem 15.2.1 is from [BK88]. A good general source on CohenMacaulay complexes is [Sta96]; Theorems 15.2.2, 15.2.3, and 15.2.4, as well as references to the original sources, can be found there. A generalization of Theorem 15.2.2 to complexes whose $k$-skeleton is Cohen-Macaulay appears in [Bjo96]. There are several additional results about $h$-vectors of Cohen-Macaulay complexes. For instance, for complexes with nontrivial automorphism groups, see [Sta96, Section III.8]; for matroid complexes, see [Sta96, Section III.3]; and for Cohen-Macaulay complexes that are $r$-colorable for $r<d$, see the references mentioned in [Sta96, Section III.4].

Cohen-Macaulay complexes are closely related to certain commutative rings [Sta96], and via this connection such complexes have also been of use in the theory of splines; see [Sta96, Section III.5] and also Chapter 45.

Theorem 15.2.5 was conjectured by Eckhoff and proved by Kalai [Kal84, Kal86].

\subsection{SIMPLICIAL POLYTOPES, SPHERES, AND MANIFOLDS}

\section{GLOSSARY}

A triangulated $\boldsymbol{d}$-ball is a simplicial complex $\Delta$ whose realization $\|\Delta\|$ is homeomorphic to the ball $\left\{x \in \mathbb{R}^{d} \mid x_{1}^{2}+\cdots+x_{d}^{2} \leq 1\right\}$. A triangulated $(\boldsymbol{d}-\mathbf{1})$ sphere is a simplicial complex whose realization is homeomorphic to the sphere $\left\{x \in \mathbb{R}^{d} \mid x_{1}^{2}+\cdots+x_{d}^{2}=1\right\}$. Equivalently, it is the boundary of a triangulated $d$-ball. Examples of triangulated $(d-1)$-spheres are given by the boundary complexes of simplicial $d$-polytopes.

A pseudomanifold is a pure simplicial complex $\Delta$ such that

(i) each face of codimension 1 is contained in precisely two maximal faces; and

(ii) the dual graph (whose vertices are the maximal faces of $\Delta$ and whose edges are the faces of codimension 1) is connected.

An Eulerian pseudomanifold is a pseudomanifold $\Delta$ such that $\Delta$ and the link of each face has the Euler characteristic of a sphere of the corresponding dimension.

A pure $(d-1)$-dimensional simplicial complex $\Delta$ is a homology manifold if it is connected and the link of each nonempty face has the Betti numbers of a sphere of the same dimension. It is a homology sphere if, in addition, $\Delta$ itself has the Betti numbers of a $(d-1)$-sphere. Examples of homology manifolds are given by triangulations of compact connected topological manifolds, i.e., spaces that are locally Euclidean. 
The cyclic d-polytope with $\boldsymbol{n}$ vertices $C_{d}(n)$ is the convex hull of any $n$ points on the moment curve in $\mathbb{R}^{d}$. (See Section 13.1.4.)

The following implications hold among these various classes, all of them strict:

$$
\begin{gathered}
\text { polytope boundary } \Rightarrow \text { sphere } \Rightarrow \text { homology sphere } \Rightarrow \\
\text { Eulerian pseudomanifold } \Rightarrow \text { pseudomanifold } \\
\text { homology sphere } \Rightarrow \text { homology manifold } \Rightarrow \text { pseudomanifold } \\
\text { homology sphere } \Rightarrow \text { Cohen-Macaulay complex }
\end{gathered}
$$

\section{PSEUDOMANIFOLDS}

The following results give the basic lower and upper bounds on $f$-vectors of pseudomanifolds.

THEOREM 15.3.1 Lower Bound Theorem

For a $(d-1)$-dimensional pseudomanifold $\Delta$ with $n$ vertices,

$$
f_{k}(\Delta) \geq \begin{cases}\left(\begin{array}{l}
d \\
k
\end{array}\right) n-\left(\begin{array}{l}
d+1 \\
k+1
\end{array}\right) k & \text { for } 1 \leq k \leq d-2 \\
(d-1) n-(d-2)(d+1) & \text { for } k=d-1\end{cases}
$$

\section{THEOREM 15.3.2 Upper Bound Theorem}

Let $\Delta$ be a (d-1)-dimensional homology manifold with $n$ vertices, such that either

(i) $d$ is even, or

(ii) $d=2 k+1$ is odd, and either $\chi(\Delta)=2$ or $\beta_{k} \leq 2 \beta_{k-1}+2 \sum_{i=0}^{k-3} \beta_{i}$.

Then $f_{k}(\Delta) \leq f_{k}\left(C_{d}(n)\right)$ for $1 \leq k \leq d-1$.

This upper bound theorem applies when the homology manifold is Eulerian (irrespective of dimension); in particular, it applies to all simplicial polytopes and spheres. By the geometric operation of "pulling vertices," one can extend this to all convex polytopes.

\section{THEOREM 15.3.3}

If $P$ is any convex $d$-polytope with $n$ vertices, then $f(P) \leq f\left(C_{d}(n)\right)$.

The given lower and upper bounds are best possible within the class of simplicial polytope boundaries. The lower bound is attained by the class of stacked polytopes (defined in Chapter 18). To make the upper bound numerically explicit, we give the formula for the $f$-vector of a cyclic polytope.

\section{THEOREM 15.3.4}

For $d \geq 2$ and $0 \leq k \leq d-1$, the number of $k$-faces of the cyclic polytope $C_{d}(n)$ with $n$ vertices is

$$
f_{k}\left(C_{d}(n)\right)=\frac{n-\delta(n-k-2)}{n-k-1} \sum_{j=0}^{\lfloor d / 2\rfloor}\left(\begin{array}{l}
n-1-j \\
k+1-j
\end{array}\right)\left(\begin{array}{c}
n-k-1 \\
2 j-k-1+\delta
\end{array}\right)
$$


where $\delta=d-2\lfloor d / 2\rfloor$. In particular,

$$
f_{d-1}\left(C_{d}(n)\right)=\left(\begin{array}{c}
n-\left\lfloor\frac{d+1}{2}\right\rfloor \\
n-d
\end{array}\right)+\left(\begin{array}{c}
n-\left\lfloor\frac{d+2}{2}\right\rfloor \\
n-d
\end{array}\right) .
$$

\section{POLYTOPES AND SPHERES}

For boundaries of simplicial $d$-polytopes and, more generally, for Eulerian pseudomanifolds, we have the following basic relations.

THEOREM 15.3.5 Dehn-Sommerville Equations

For d-dimensional Eulerian pseudomanifolds,

$$
h_{i}=h_{d-i} \quad \text { for all } 0 \leq i \leq d .
$$

These equations give a complete description of the linear span of all $f$-vectors of $d$-polytopes (equivalently, $(d-1)$-spheres). (The affine span is defined by including the relation $h_{0}=1$.)

One consequence of the Dehn-Sommerville equations is the following relation between the $h$-vector of a triangulated ball $K$ and the $g$-vector of its boundary $\partial K$.

\section{THEOREM 15.3.6}

For a triangulated $d$-ball $K$ and its boundary $(d-1)$-sphere $\partial K$,

$$
g_{i}(\partial K)=h_{i}(K)-h_{d+1-i}(K) \quad \text { for } i \geq 1 \text {. }
$$

A complete characterization of the $f$-vectors of simplicial (and, by duality, simple) convex polytopes is given in terms of the $h$-vector and $g$-vector.

THEOREM 15.3.7 $g$-Theorem

A nonnegative integer vector $h=\left(h_{0}, \ldots, h_{d}\right)$ is the $h$-vector of a simplicial convex polytope if and only if

(i) $h_{i}=h_{d-i}$, and

(ii) $\left(g_{0}, \ldots, g_{\lfloor d / 2\rfloor}\right)$ is an $M$-sequence.

One consequence of (ii) is that $g_{i} \geq 0$. For centrally symmetric polytopes, we get a better lower bound.

\section{THEOREM 15.3.8}

For centrally symmetric simplicial d-polytopes,

$$
g_{i}=h_{i}-h_{i-1} \geq\left(\begin{array}{l}
d \\
i
\end{array}\right)-\left(\begin{array}{c}
d \\
i-1
\end{array}\right) \quad \text { for } i \leq\lfloor d / 2\rfloor
$$

\section{COMMENTS}

The Lower Bound Theorem 15.3.1 is due to Kalai and Gromov in the generality given here; see [Kal87] including the note added in proof. The $k=d-1$ case had 
earlier been done by Klee and the case of polytope boundaries by Barnette. See [Kal87] for a discussion of the history of this result.

The Upper Bound Theorem 15.3.2 is due to Novik [Nov95]. The case of polytopes (Theorem 15.3.3) was first proved by McMullen (see [MS71]), and extended to spheres by Stanley (see [Sta96]). The computation of the $f$-vector of the cyclic polytope can be found in [Gru67, Sections 4.7.3 and 9.6.1] or [MS71].

The Dehn-Sommerville equations for polytopes are classical; proofs can be found in [Gru67, Sta86, Zie95]. The extension to Eulerian pseudomanifolds is due to Klee [Kle64]; an equivariant version appears in [Bar92]. The $g$-theorem was conjectured by McMullen and proved by Billera, Lee, and Stanley [BL81, Sta80]. More recently, another proof of the necessity of these conditions was given by McMullen [McM93]. It is not known whether the second condition of Theorem 15.3.7 holds for general triangulated spheres. The $g$-theorem has a convenient reformulation as a one-to-one correspondence (via matrix multiplication) between $f$-vectors of simplicial polytopes and $M$-sequences, see [Bjo87, Zie95]. Theorem 15.3.8 was proved by Stanley [Sta87a].

The study of $f$-vectors of unbounded polyhedra can be approached by studying the $f$-vectors of polytope pairs $(P, F)$, where $P$ is a polytope and $F$ is a maximal face of $P$. See [BL93] for a summary of such results.

\subsection{CELL COMPLEXES}

\section{GLOSSARY}

Convex polytopes and faces of such are defined in Chapter 13.

A polyhedral complex $\Gamma$ is a finite collection of convex polytopes in $\mathbb{R}^{n}$ such that (i) if $\pi \in \Gamma$ and $\sigma$ is a face of $\pi$ then $\sigma \in \Gamma$, and (ii) if $\pi, \sigma \in \Gamma$ and $\pi \cap \sigma \neq \emptyset$ then $\pi \cap \sigma$ is a face of both. The space of $\Gamma$ is $\|\Gamma\|=\bigcup_{\pi \in \Gamma} \pi$, a subspace of $\mathbb{R}^{n}$. Examples of polyhedral complexes are given by boundary complexes $\partial P$ of convex polytopes $P$ (i.e., the collection of all proper faces). A geometric simplicial complex (defined in Section 15.1) is a polyhedral complex all whose cells are simplices. A cubical complex is a polyhedral complex all whose cells are (combinatorially isomorphic to) cubes.

A regular cell complex $\Gamma$ is a family of closed balls (homeomorphs of $\{x \in$ $\left.\mathbb{R}^{j}|| x \mid \leq 1\right\}$ ) in a Hausdorff space $\|\Gamma\|$ such that (i) the interiors of the balls partition $\|\Gamma\|$ and (ii) the boundary of each ball in $\Gamma$ is a union of other balls in $\Gamma$. The members of $\Gamma$ are called (closed) cells or faces. The dimension of a cell is its topological dimension and $\operatorname{dim} \Gamma=\max _{\sigma \in \Gamma} \operatorname{dim} \sigma$.

A regular cell complex has the intersection property if, whenever the intersection of two cells is nonempty, then this intersection is also a cell in the complex. Polyhedral complexes are examples of regular cell complexes with the intersection property. Regular cell complexes with the intersection property can be reconstructed up to homeomorphism from the corresponding "abstract" complex consisting of the family of vertex sets of its cells.

For a regular cell complex $\Gamma$, let $f_{i}$ be the number of $i$-dimensional cells, and let $\beta_{i}=\operatorname{dim}_{\mathbb{Q}} \widetilde{H}_{i}(\|\Gamma\|, \mathbb{Q})$. The latter denotes $i$-dimensional reduced singular 
homology with rational coefficients of the space $\|\Gamma\|$; see [Mun84, Spa66] for explanations of this concept. Then we have the $f$-vector $f=\left(f_{0}, f_{1}, \ldots\right)$ and the Betti sequence $\beta=\left(\beta_{0}, \beta_{1}, \ldots\right)$ of $\Gamma$. These definitions generalize those previously given in the simplicial case.

\section{BASIC $f$-VECTOR RELATIONS}

Among the classes of complexes

- simplicial complexes

- polyhedral complexes

- regular cell complexes with the intersection property

- regular cell complexes

each is a subclass of its successor. Thus one may wonder how many of the relations for $f$-vectors of simplicial complexes given in Sections 15.1-15.3 can be extended to these broader classes of complexes. Also, what new phenomena (not visible in the simplicial case) arise? Some answers will be given in this section and the following one, but current knowledge is quite fragmentary. We begin here with the most general relations.

\section{THEOREM 15.4.1}

$\left(f_{0}, \ldots, f_{d}\right)$ is the f-vector of a d-dimensional regular cell complex if and only if $f_{d} \geq 1$ and $f_{i} \geq 2$ for all $0 \leq i<d$.

\section{THEOREM 15.4.2}

$f$ is the $f$-vector of a regular cell complex with the intersection property if and only if $f$ is a $K$-sequence. let

Let $\beta=\left(\beta_{0}, \beta_{1}, \ldots\right) \in \mathbb{N}^{(\infty)}$ be fixed, and for every sequence $f=\left(f_{0}, f_{1}, \ldots\right)$

$$
\chi_{k-1}=\sum_{j \geq k}(-1)^{j-k}\left(f_{j}-\beta_{j}\right) \quad \text { for } k \geq 0 .
$$

\section{THEOREM 15.4.3}

$\left(f_{0}, \ldots, f_{d}\right)$ is the $f$-vector of a d-dimensional regular cell complex with Betti sequence $\beta$ if and only if $\chi_{-1}=1$ and $\chi_{k} \geq 1$ for $0 \leq k<d$.

\section{THEOREM 15.4.4}

For $f \in \mathbb{N}^{(\infty)}$ the following are equivalent:

(i) $f$ is the $f$-vector of a regular cell complex with the intersection property and with Betti sequence $\beta$;

(ii) $\chi_{-1}=1$ and $\partial_{k+1}\left(\chi_{k}+\beta_{k}\right) \leq \chi_{k-1}$ for all $k \geq 1$.

These results show that the $f$-vectors of regular cell complexes (with or without Betti number constraints) are a lot more general than the $f$-vectors of simplicial complexes, but that the two classes of $f$-vectors agree in the presence of the intersection property. 


\section{COMMENTS}

Regular cell complexes are known as regular $C W$ complexes in the topological literature [LW69]. The non-regular CW complexes offer an even more general class of cell complexes [LW69, Mun84, Spa66], but there is very little one can say about $f$-vectors in that generality. See [BLS ${ }^{+} 93$, Section 4.7] for a detailed discussion of regular cell complexes from a combinatorial point of view.

For the results of this section see [BK88, BK91, BK89]. A characterization of $f$-vectors of (cubical) subcomplexes of a cube can be found in [Lin71], and of regular cell decompositions of spheres in [Bay88].

\subsection{GENERAL POLYTOPES AND SPHERES}

\section{GLOSSARY}

A flag of faces in a (polyhedral) $(d-1)$-complex $\Delta$ is a chain $F_{1} \subsetneq F_{2} \subsetneq \cdots \subsetneq F_{k}$ in $\Delta$. It is an $S$-flag if

$$
S=\left\{\operatorname{dim} F_{1}, \ldots, \operatorname{dim} F_{k}\right\} \subseteq\{0,1, \ldots, d-1\} .
$$

If $f_{S}=f_{S}(\Delta)$ denotes the number of $S$-flags in $\Delta$, then the function $S \mapsto f_{S}$, $S \subseteq\{0,1, \ldots, d-1\}$, is called the flag $f$-vector of $\Delta$.

If

$$
h_{S}=\sum_{T \subseteq S}(-1)^{|S|-|T|} f_{T},
$$

then the function $S \mapsto h_{S}, S \subseteq\{0,1, \ldots, d-1\}$, is called the flag h-vector.

For $S \subseteq\{0, \ldots, d-1\}$ and noncommuting symbols $\boldsymbol{a}$ and $\boldsymbol{b}$, let $u_{S}=u_{0} u_{1} \cdots u_{d-1}$ be the $\boldsymbol{a} \boldsymbol{b}$-word defined by $u_{i}=\boldsymbol{a}$ if $i \notin S$ and $u_{i}=\boldsymbol{b}$ otherwise. When $\Delta$ is spherical (or, more generally, Eulerian), then the $\boldsymbol{a b}$-polynomial $\sum h_{S} u_{S}$ is also a polynomial in $\boldsymbol{c}=\boldsymbol{a}+\boldsymbol{b}$ and $\boldsymbol{d}=\boldsymbol{a b}+\boldsymbol{b} \boldsymbol{a}$. (Note that the degree of $\boldsymbol{c}$ is 1 and the degree of $\boldsymbol{d}$ is 2.) The resulting $\boldsymbol{c d}$-polynomial

$$
\sum h_{S} u_{S}=\sum \phi_{w} w
$$

where the right-hand sum is over all $\boldsymbol{c d}$-words $w$ of degree $d$, is called the $\boldsymbol{c d}$ index of $\Delta$. For 2- and 3-polytopes, the $\boldsymbol{c d}$-index is $\boldsymbol{c}^{2}+\left(f_{0}-2\right) \boldsymbol{d}$ and $\boldsymbol{c}^{3}+$ $\left(f_{0}-2\right) \boldsymbol{d} \boldsymbol{c}+\left(f_{2}-2\right) \boldsymbol{c} \boldsymbol{d}$, respectively.

For any convex $d$-polytope $P$, we define the toric h-vector and toric g-vector recursively by $h(P, x)=\sum_{i=0}^{d} h_{i} x^{d-i}$ and $g(P, x)=\sum_{i=0}^{\lfloor d / 2\rfloor} g_{i} x^{i}$, where $g_{i}=$ $h_{i}-h_{i-1}$ and the following relations hold:

(i) $g(\emptyset, x)=h(\emptyset, x)=1$, and

(ii) $h(P, x)=\sum_{G \text { face of } P, G \neq P} g(G, x)(x-1)^{d-1-\operatorname{dim} G}$.

(Compare Chapter 14.4.2, where this toric $h$-vector is defined for any polyhedral complex. In the notation given there, we have defined $h$ and $g$ for the complex $\partial P$.) When $P$ is simplicial, this definition coincides with that of the usual $h$ vector, as defined in Section 15.2.

A rational polytope is one whose vertices all have rational coordinates. 
A cubical polytope is one that has a cubical boundary complex. For any cubical $(d-1)$-complex with $f$-vector $\left(f_{0}, \ldots, f_{d-1}\right)$, define the cubical h-vector $h^{c}=$ $\left(h_{0}^{c}, \ldots, h_{d}^{c}\right)$ by

$$
h_{i}^{c}=(-1)^{i} 2^{d-1}+\sum_{j=1}^{i}(-1)^{i-j} 2^{j-1} f_{j-1} \sum_{k=0}^{i-j}\left(\begin{array}{c}
d-j \\
k
\end{array}\right) \quad \text { for } i=0, \ldots, d \text {. }
$$

The cubical g-vector $g^{e}=\left(g_{0}^{e}, \ldots, g_{\lfloor d / 2\rfloor}^{e}\right)$ is defined by $g_{0}^{c}=h_{0}^{c}=2^{d-1}$ and $g_{i}^{c}=h_{i}^{c}-h_{i-1}^{c}$ for $i \geq 1$.

An Eulerian polyhedral complex is one whose first barycentric subdivision is an Eulerian pseudomanifold. Examples are boundary complexes of polytopes and spherical polyhedral complexes, i.e., those whose underlying space is homeomorphic to a sphere.

A (central) hyperplane arrangement is a collection $\mathcal{H}$ of $n$ linear hyperplanes in $\mathbb{R}^{d}$, given by normal vectors $x_{1}, \ldots, x_{n}$ (see Section 6.1 .3 ). The arrangement is essential if the normals $x_{i}$ span $\mathbb{R}^{d}$. The associated zonotope is the Minkowski sum of the $n$ line segments $\left[-x_{i}, x_{i}\right]$, i.e., $Z=\left\{\sum \lambda_{i} x_{i} \mid-1 \leq \lambda_{i} \leq 1\right\}$ (see Section 13.1.4).

\section{LINEAR RELATIONS}

We give the linear relations on the invariants defined above that are known to hold for all boundary complexes of polytopes and, more generally, for all Eulerian polyhedral complexes.

\section{THEOREM 15.5.1}

For (d-1)-dimensional Eulerian polyhedral complexes, the following relations always hold for the flag $h$, the toric $h$, and the flag $f$ :

(i) $h_{S}=h_{\{0, \ldots, d-1\} \backslash S}$ for all $S \subseteq\{0, \ldots, d-1\}$;

(ii) $h_{i}=h_{d-i} \quad$ for $0 \leq i \leq d$; and

(iii) $\sum_{j=i+1}^{k-1}(-1)^{j-i-1} f_{S \cup\{j\}}=\left(1-(-1)^{k-i-1}\right) f_{S} \quad$ whenever $i, k \in S \cup\{-1, d\}$ with $i \leq k-2$ and $S \cap\{i+1, \ldots, k-1\}=\emptyset$.

It is known that the relations in Theorem 15.5.1(iii), the generalized DehnSommerville equations, completely describe the linear span of all flag $f$-vectors of Eulerian complexes, and so they imply those in (i). Since the toric $h$ is known to be a linear function of the flag $f$, they imply those in (ii) as well. The linear span of flag $f$-vectors has dimension $e_{d}$, where $e_{d}$ is the $d^{\text {th }}$ Fibonacci number (defined by the recurrence $\left.e_{d}=e_{d-1}+e_{d-2}, e_{0}=e_{1}=1\right)$. There are $e_{d} \boldsymbol{c d}$-words of degree $d$. Furthermore, the coefficients $\phi_{w}$ of the $\boldsymbol{c d}$-index, considered as linear expressions in the $f_{S}$, form a linear basis for the span of flag $f$-vectors of $d$-polytopes. The affine span of all flag $f$-vectors is defined by including the relation $f_{\emptyset}=1$.

For cubical polytopes and spheres, the cubical $h$-vector satisfies the analogue of the Dehn-Sommerville equations.

\section{THEOREM 15.5.2}

For cubical d-polytopes and cubical $(d-1)$-spheres,

$$
h_{i}^{c}=h_{d-i}^{c} \quad \text { for all } 0 \leq i \leq d .
$$


These give all linear relations satisfied by $f$-vectors of cubical polytopes and spheres. The cubical $h$-vector satisfies, as well, the equations of Theorem 15.3.6, linking the $h$ of a cubical ball to the $g$ of its boundary sphere.

\section{LINEAR INEQUALITIES}

Some linear inequalities that hold for flag $f$-vectors of all polytope boundaries are given in this section. The list is not thought to be complete, although there are no conjectures for what the complete set might be.

For a Cohen-Macaulay polyhedral complex, i.e., one whose first barycentric subdivision is a Cohen-Macaulay simplicial complex, the flag $h$ is always nonnegative.

\section{THEOREM 15.5.3}

For a Cohen-Macaulay polyhedral $(d-1)$-complex $\Gamma$,

$$
h_{S}(\Gamma) \geq 0 \quad \text { for all } S \subseteq\{0, \ldots, d-1\} .
$$

For general convex polytopes, we also have nonnegativity of the $\boldsymbol{c d}$-index.

\section{THEOREM 15.5.4}

For a convex d-polytope $P, \phi_{w} \geq 0$ for all cd-words $w$ of degree $d$.

For rational convex polytopes, it is known, further, that the toric $h$ is unimodal.

\section{THEOREM 15.5.5}

For a rational convex d-polytope, $g_{i} \geq 0$ for $i \leq\lfloor d / 2\rfloor$.

Related to this is the following nonlinear inequality holding between the $g$ vectors of a polytope $P$ and any of its faces $F$. We denote by $P / F$ the $\operatorname{lin} \boldsymbol{k}$ of $F$ in $P$, i.e., the polytope whose lattice of faces is (isomorphic to) the interval $[F, P]$ in the face lattice of $P$.

\section{THEOREM 15.5.6}

For a rational polytope $P$ and any face $F$, we have the polynomial inequality

$$
g(P, t)-g(F, t) g(P / F, t) \geq 0,
$$

i.e., all coefficients of this polynomial are nonnegative.

Finally, we have the following lower bound for the number of vertices of polytopes with no triangular faces (this includes the class of cubical polytopes).

THEOREM 15.5.7

A d-polytope with no triangular 2 -face has at least $2^{d}$ vertices.

\section{HYPERPLANE ARRANGEMENTS AND ZONOTOPES}

An essential hyperplane arrangement $\mathcal{H}$ defines a decomposition of $\mathbb{R}^{d}$ into polyhedral cones (as in Section 6.1.3). This decomposition $\Gamma_{\mathcal{H}}$, a regular cell complex if in- 
tersected with the unit sphere, has a flag $f$-vector dual to that of its associated zonotope $Z$, in the sense that $f_{S}\left(\Gamma_{\mathcal{H}}\right)=f_{d-S}(Z)$, where $S=\left\{i_{1}, \ldots, i_{k}\right\} \subseteq\{1, \ldots, d\}$ and $d-S=\left\{d-i_{k}, \ldots, d-i_{1}\right\}$.

\section{THEOREM 15.5.8}

The flag $f$-vector of an arrangement (or zonotope) depends only on the matroid (linear dependency structure) of the underlying point configuration $\left\{x_{1}, \ldots, x_{n}\right\}$.

Although a fairly special subclass of polytopes, the zonotopes nonetheless are varied enough to carry all the linear information carried by flag numbers of general polytopes.

\section{THEOREM 15.5.9}

The flag f-vectors of zonotopes (and thus of hyperplane arrangements) satisfy the generalized Dehn-Sommerville equations, and no other linear relations not implied by these.

When it comes to linear inequalities, however, the difference between zonotopes and general polytopes emerges. The following result has the most direct interpretation when it is stated for arrangements, where it bounds the average number of $S=\left\{i_{1}, \ldots, i_{k}\right\}$-flags in an $i_{k}$-face by the number of $S$-flags in an $i_{k}$-cube.

\section{THEOREM 15.5.10}

For a hyperplane arrangement $\mathcal{H}$ in $\mathbb{R}^{d}$ and $S=\left\{i_{1}, \ldots, i_{k}\right\} \subseteq\{1, \ldots, d\}$ with $k \geq 2$,

$$
\frac{f_{S}\left(\Gamma_{\mathcal{H}}\right)}{f_{i_{k}}\left(\Gamma_{\mathcal{H}}\right)}<\left(\begin{array}{c}
i_{k} \\
i_{1}, i_{2}-i_{1}, \ldots, i_{k}-i_{k-1}
\end{array}\right) 2^{i_{k}-i_{1}}
$$

There is a straightforward reformulation of Theorem 15.5.10 for zonotopes that is easily seen not to be valid for all polytopes.

\section{GENERAL 3- AND 4-POLYTOPES}

We describe here the situation for flag $f$-vectors of 3 - and 4-polytopes. The equations in Theorem 15.5.1(iii) reduce consideration to $\left(f_{0}, f_{2}\right)$ when $d=3$ and to $\left(f_{0}, f_{1}, f_{2}, f_{02}\right)$ when $d=4$.

\section{THEOREM 15.5.11}

For 3-polytopes, the following is known about the vector $\left(f_{0}, f_{2}\right)$.

(i) An integer vector $\left(f_{0}, f_{2}\right)$ is the $f$-vector of a 3 -polytope if and only if $f_{0} \leq$ $2 f_{2}-4$ and $f_{2} \leq 2 f_{0}-4$.

(ii) An integer vector $\left(f_{0}, f_{2}\right)$ is the $f$-vector of a cubical 3-polytope if and only if $f_{2}=f_{0}-2, f_{0} \geq 8$, and $f_{0} \neq 9$.

(iii) If $\left(f_{0}, f_{2}\right)=\left(f_{0}(Z), f_{2}(Z)\right)$ for a 3 -zonotope $Z$, then $f_{0}$ and $f_{1}$ are both even integers, $f_{0} \leq 2 f_{2}-4$, and $f_{2} \leq f_{0}-2$.

For 4-polytopes, much less is known. 


\section{THEOREM 15.5.12}

Flag $f$-vectors $\left(f_{0}, f_{1}, f_{2}, f_{02}\right)$ of 4-polytopes satisfy the following inequalities.

(i) $f_{02} \geq 3 f_{2}$

(ii) $f_{02} \geq 3 f_{1}$

(iii) $f_{02}+f_{1}+10 \geq 3 f_{2}+4 f_{0}$

(iv) $6 f_{1} \geq 6 f_{0}+f_{02}$

(v) $f_{0} \geq 5$

(vi) $f_{0}+f_{2} \geq f_{1}+5$

(vii) $2\left(f_{02}-3 f_{2}\right) \leq\left(\begin{array}{c}f_{0} \\ 2\end{array}\right)$

(viii) $2\left(f_{02}-3 f_{1}\right) \leq\left(\begin{array}{c}f_{2}-f_{1}+f_{0} \\ 2\end{array}\right)$

(ix) $f_{02}-4 f_{2}+3 f_{1}-2 f_{0} \leq\left(\begin{array}{c}f_{\circ} \\ 2\end{array}\right)$

(x) $f_{02}+f_{2}-2 f_{1}-2 f_{0} \leq\left(\begin{array}{c}f_{2}-f_{1}+f_{0} \\ 2\end{array}\right)$.

It is not known, for example, whether (i)-(vi) give all linear inequalities holding for flag $f$-vectors of 4-polytopes.

\section{COMMENTS}

It is thought that the best route to an eventual characterization of $f$-vectors of general polytopes lies in an understanding of their flag $f$-vectors. The latter inherit many of the algebraic properties of $f$-vectors of simplicial polytopes that led to their characterization.

The relations in Theorem 15.5.1 hold more generally for the case of enumeration of chains in Eulerian posets; see the article by Stanley in [BMS ${ }^{+94]}$. The relations in Theorem 15.5.1(iii) are proved in [BB85]. An expression for the toric $h$ in terms of the flag $f$, as well as a discussion of the convolution product (originally due to Kalai), can be found in the article by Bayer in $\left[\mathrm{BMS}^{+} 94\right]$. The article by Kalai in the same volume contains an extensive discussion of $g$-vectors for both simplicial and general polytopes. The form of the cubical Dehn-Sommerville equations given in Theorem 15.5.2 appeared in [Adi97].

Theorem 15.5.4 holds as well for certain shellable spheres (see [Sta96, Section III.4]). Theorem 15.5.3 can also be found in [Sta96, Theorem III.4.4] (where $h_{S}$ is denoted $\left.\beta(S)\right)$. Theorem 15.5.5 appears in [Sta87b]. Theorem 15.5.7 is due to Blind and Blind [BB90]. There is a notion of convolution product of flag $f$ numbers that can be used to produce new linear inequalities from given ones; see [BL93, Section 3.10].

Note that Theorem 15.5.6, due to Braden and MacPherson [BM96], gives a connection between the $g$-vector of a polytope $P$ and that of one of its faces. This is an example of a "monotonicity theorem" related to face numbers. For similar theorems relating $h$-vectors of subcomplexes and subdivisions of a simplicial complex $\Delta$, see Sections III.9-10 of [Sta96] and the references given there.

For the fact that the flag $f$-vector of a zonotope or arrangement (or, more generally, of an oriented matroid) depends only on the underlying matroid see $\left[\mathrm{BLS}^{+} 93\right.$, Cor. 4.6.3]. That the only linear relations satisfied by zonotopes are 
the generalized Dehn-Sommerville equations of Theorem 15.5.1(iii) is proved in [BER96]. Theorem 15.5.10 is due to Varchenko for the case $k=2$ (see [BLS ${ }^{+}$93, Proposition 4.6.9]) and to Liu [Liu95, Theorem 4.8.2] in the form given here.

Theorem 15.5.11(i) can be found in [Gru67, Section 10.3]; 15.5.11(ii) appears in dual form (for 4-valent 3-polytopes) in [Bar83]; 15.5.11(iii) can be derived using the methods of [Gru67, Section 18.2] (see also [BER96]). Theorem 15.5.12 can be found in [Bay87].

\subsection{OPEN PROBLEMS}

\section{PROBLEM 15.6.1}

Characterize the $f$-vectors of triangulations of the $(d-1)$-sphere. It has been conjectured that the conditions of the $g$-theorem provide the answer.

\section{PROBLEM 15.6.2}

Characterize the $f$-vectors of triangulations of the $d$-ball.

\section{PROBLEM 15.6.3}

Characterize the $f$-vectors of triangulations of the d-torus. It is known that $f$ (2-torus) $=\{(n, 3 n, 2 n) \mid n \geq 7\}$, but the question is open for $d \geq 3$.

\section{PROBLEM 15.6.4}

Characterize the $f$-vectors of $d$-polytopes. The answer is known for $d \leq 3$ (Theorem 15.5.11(i)), but for $d \geq 4$ there is not even a conjectured answer.

\section{PROBLEM 15.6.5 I. Bárány}

Does there exist a constant $c_{d}>0$ such that $f_{i} \geq c_{d} \cdot \min \left\{f_{0}, f_{d-1}\right\}$ for all d-polytopes and all $i$ ? Will $c_{d}=1$ do?

\section{PROBLEM 15.6.6}

Characterize the f-vectors of centrally symmetric d-polytopes. The question is open in the simplicial as well as in the general case. Even an upper bound conjecture in the simplicial and centrally symmetric case is missing.

\section{PROBLEM 15.6.7 Conjecture of G. Kalai}

The total number of faces (counting $P$ but not $\emptyset$ ) of a centrally symmetric convex d-polytope $P$ is $\geq 3^{d}$. (Verified in the simplicial case as a consequence of Theorem 15.3.8.)

\section{PROBLEM 15.6.8}

The clique complex of a graph is the collection of vertex sets of all its cliques (complete induced subgraphs). Characterize the $f$-vectors of clique complexes. 
PROBLEM 15.6.9 G. Kalai

Is the $f$-vector of an $(r-1)$-dimensional clique complex the $f$-vector of an $r$-colorable complex?

PROBLEM 15.6.10 Conjecture of Charney and Davis [Sta96, p. 100] Let $\left(g_{0}, \ldots, g_{k}\right)$ be the $g$-vector of a clique complex homeomorphic to the sphere $S^{2 k-1}$. Then $g_{k}-g_{k-1}+\ldots+(-1)^{k} g_{0} \geq 0$.

PROBLEM 15.6.11 Conjecture of Stanley [Sta96, p. 102] Every coefficient $\phi_{w}$ of the cd-index of a sphere is nonnegative.

PROBLEM 15.6.12 Adin [Adi97] (The case $i=1$ is implied by Theorem 15.5.7.) The generalized lower bound conjecture for cubical d-polytopes and d-spheres: $g_{i}^{c} \geq 0$ for $i \leq\lfloor d / 2\rfloor$. This has been shown to be the best possible set of linear inequalities for cubical d-spheres [BBC96]. More generally, characterize $f$-vectors of cubical polytopes.

\section{PROBLEM 15.6.13}

Characterize the flag $f$-vectors of polytopes and of zonotopes. In particular, determine a complete set of linear inequalities holding for flag $f$-vectors of polytopes and of zonotopes.

\section{PROBLEM 15.6.14}

Characterize (toric) h-vectors of general polytopes.

\subsection{SOURCES AND RELATED MATERIAL}

\section{FURTHER READING}

Surveys of $f$-vector theory are given in [BL93, Bjo87, BK89, KK95, Sta85]. Books treating $f$-vectors (among other things) include [And87, $\mathrm{BMS}^{+} 94$, Gru67, MS71, Sta96, Zie95].

\section{RELATED CHAPTERS}

Chapter 6: Oriented matroids

Chapter 13: Basic properties of convex polytopes

Chapter 14: Subdivisions and triangulations of polytopes

Chapter 45: Splines and geometric modeling

\section{REFERENCES}

[Adi97] R.M. Adin. A new cubical h-vector. Discrete Math. (to appear).

[And87] I. Anderson. Combinatorics of Finite Sets. Clarendon Press, Oxford, 1987. 
[BBC96] E.K. Babson, L.J. Billera, and C. Chan. Neighborly cubical spheres and a cubical lower bound conjecture. Israel J. Math. (to appear).

[Bar83] D.W. Barnette. Map Coloring, Polyhedra, and the Four Color Theorem. Dolciani Mathematics Expositions No. 8, Mathematical Association of America, Washington, 1983.

[Bar92] A.I. Barvinok. On equivariant generalization of Dehn-Sommerville equation. Europ. $J$. Combin. 13 (1992), 419-428.

[Bay87] M.M. Bayer. The extended f-vectors of 4-polytopes. J. Combin. Theory. Ser. A 44 (1987), 141-151.

[Bay88] M.M. Bayer. Barycentric subdivisions. Pacific J. Math. 135 (1988), 1-16.

[BB85] M.M. Bayer and L.J. Billera. Generalized Dehn-Sommerville relations for polytopes, spheres and Eulerian partially ordered sets. Invent. Math. 79 (1985), 143-157.

[BL93] M.M. Bayer and C.W. Lee. Combinatorial aspect of convex polytopes. In Handbook of Convex Geometry (P. Gruber and J.M. Wills, eds.), North-Holland, Amsterdam, 1993, pp. $485-534$.

[BER96] L.J. Billera, R. Ehrenborg, and M. Readdy. The $c d$-index of zonotopes and arrangements. Rota Festschrift (submitted).

[BL81] L.J. Billera and C.W. Lee. A proof of the sufficiency of McMullen's conditions for $f$-vectors of simplicial polytopes. J. Combin. Theory Ser. A 31 (1981), 237-255.

[BMS ${ }^{+}$94] T. Bisztriczky, P. McMullen, R. Schneider, and A. Ivić Weiss, eds.. Polytopes: Abstract, Convex and Computational. Series C: Mathematical and Physical Sciences, Vol. 440, Kluwer, Dordrecht, 1994.

[Bjo87] A. Björner. Face numbers of complexes and polytopes. In Proceedings of the International Congress of Mathematicians, Berkeley, 1986, Amer. Math. Soc., Providence, 1987, pp. 1408-1418.

[Bjo96] A. Björner. Nonpure shellability, $f$-vectors, subspace arrangements and complexity. In Formal Power Series and Algebraic Combinatorics (L.J. Billera, C. Greene, R. Simion, and R. Stanley, eds.), DIMACS Series in Discrete Math. and Theor. Computer Science, Amer. Math. Soc., Providence, 1996, pp. 25-53.

[BK88] A. Björner and G. Kalai. An extended Euler-Poincaré theorem. Acta Math. 161 (1988), 279-303.

[BK89] A. Björner and G. Kalai. On $f$-vectors and homology. In Combinatorial Mathematics: Proc. 3rd Intern. Conf., New York, 1985 (G. Bloom, R. Graham, and J. Malkevitch, eds.), Annals New York Academy of Sciences, Vol. 555, 1989, pp. 63-80.

[BK91] A. Björner and G. Kalai. Extended Euler-Poincaré relations for cell complexes. In $A p$ plied Geometry and Discrete Mathematics (The Victor Klee Festschrift) (P. Gritzmann and B. Sturmfels, eds.), Amer. Math. Soc., Providence, 1991, pp. 81-89.

$\left[\mathrm{BLS}^{+} 93\right] \quad$ A. Björner, M. LasVergnas, B. Sturmfels, N. White, and G.M. Ziegler. Oriented Matroids. Encyclopedia of Math. 46, Cambridge Univ. Press, 1993.

[BB90] G. Blind and R. Blind. Convex polytopes without triangular faces. Israel Jour. Math. 71 (1990), 129-134.

[BM96] T.C. Braden and R. MacPherson. Intersection homology of toric varieties and a conjecture of Kalai. (In preparation.)

[FFK88] P. Frankl, Z. Füredi, and G. Kalai. Shadows of colored complexes. Math. Scand. 63 (1988), 169-178.

[FK96] E. Friedgut and G. Kalai. Every monotone graph property has a sharp threshold. Proc. Amer. Math. Soc. (to appear). 
[Gru67] B. Grünbaum. Convex Polytopes. Interscience/Wiley, New York, 1967. (New edition soon to appear, Springer-Verlag.)

[Hib89] T. Hibi. What can be said about pure O-sequences? J. Combin. Theory Ser. A 50 (1989), 319-322.

[Kal84] G. Kalai. A characterization of $f$-vectors of families of convex sets in $\mathbb{R}^{d}$. Part I: Necessity of Eckhoff's conditions. Israel J. Math. 48 (1984), 175-195.

[Kal86] G. Kalai. A characterization of $f$-vectors of families of convex sets in $\mathbb{R}^{d}$. Part II: Sufficiency of Eckhoff's conditions. J. Combin. Theory Ser. A 41 (1986), 167-188.

[Kal87] G. Kalai. Rigidity and the lower bound theorem 1. Invent. Math. 88 (1987), 125-151.

[Kle64] V. Klee. A combinatorial analogue of Poincaré's duality theorem. Canad. J. Math. 16 (1964), 517-531.

[KK95] V. Klee and P. Kleinschmidt. Convex polytopes and related complexes. In Handbook of Combinatorics (R. Graham, M. Grötschel, and L. Lovász, eds.), North-Holland, Amsterdam, 1995, pp. 875-917.

[Lin71] B. Lindström. The optimal number of faces in cubical complexes. Ark. Mat. 8 (1971), 245-257.

[Liu95] N. Liu. Algebraic and Combinatorial Methods for Face Enumeration in Polytopes. Ph.D. Thesis, Cornell University, Ithaca, 1995.

[LW69] A.T. Lundell and S. Weingram. The Topology of $C W$ Complexes. Van Nostrand, New York, 1969.

[McM93] P. McMullen. On simple polytopes. Invent. Math. 113 (1993), 419-444.

[MS71] P. McMullen and G.C. Shephard. Convex Polytopes and the Upper Bound Conjecture. LMS Lecture Notes, Vol. 3, Cambridge Univ. Press, London, 1971.

[Mun84] J.R. Munkres. Elements of Algebraic Topology. Addison-Wesley, Reading, 1984.

[Nov95] I. Novik. Upper bound theorems for simplicial manifolds. Preprint, 1995.

[Spa66] E.H. Spanier. Algebraic Topology. McGraw-Hill, New York, 1966.

[Sta80] R.P. Stanley. The number of faces of simplicial convex polytopes. Adv. Math. 35 (1980), 236-238.

[Sta85] R.P. Stanley. The number of faces of simplicial polytopes and spheres. In Discrete Geometry and Convexity, (J.E. Goodman, E. Lutwak, J. Malkevitch, and R. Pollack, eds.), Annals New York Academy of Sciences, Vol. 440, 1985, pp. 212-223.

[Sta86] R.P. Stanley. Enumerative Combinatorics, Vol. I. Wadsworth, Monterey, 1986.

[Sta87a] R.P. Stanley. On the number of faces of centrally-symmetric simplicial polytopes. Graphs Combin. 3 (1987), 55-66.

[Sta87b] R.P. Stanley. Generalized h-vectors, intersection cohomology of toric varieties, and related results. In Commutative Algebra and Combinatorics (M. Nagata and H. Matsumura, eds.), Advanced Studies in Pure Mathematics 11, Kinokuniya, Tokyo and North-Holland, Amsterdam/New York, 1987, pp. 187-213.

[Sta96] R.P. Stanley. Combinatorics and Commutative Algebra, 2nd Ed. Progress in Mathematics 41, Birkhäuser, Boston, 1996.

[Zie95] G.M. Ziegler. Lectures on Polytopes. Graduate Texts in Mathematics 152. SpringerVerlag, New York, 1995. 